\title{
Optical Injection-Locking-Based Pump Recovery for Phase-Sensitively Amplified Links
}

\author{
Samuel L.I. Olsson ${ }^{1}$, Bill Corcoran ${ }^{1}$, Carl Lundström ${ }^{1}$, Ekawit Tipsuwannakul ${ }^{1}$, Stylianos Sygletos ${ }^{2}$, \\ Andrew D. Ellis ${ }^{2}$, Zhi Tong ${ }^{1, \dagger}$, Magnus Karlsson ${ }^{1}$, Peter A. Andrekson ${ }^{1}$ \\ ${ }^{1}$ Photonics Laboratory, Department of Microtechnology and Nanoscience, Chalmers University of Technology, SE-412 96, Göteborg, Sweden \\ ${ }^{2}$ Tyndall National Institute and Department of Physics, University College Cork, Ireland \\ ${ }^{\dagger}$ Now at: Department of Electrical and Computer Engineering, University of California San Diego, La Jolla, CA 92093, USA \\ E-mail: samuel.olsson@chalmers.se
}

\begin{abstract}
An injection-locking-based pump recovery system for phase-sensitively amplified links is proposed and studied experimentally. Measurements with 10 Gbaud DQPSK signals show penalty-free recovery of $0.8 \mathrm{GHz}$ FWHM bandwidth pump with $63 \mathrm{~dB}$ overall amplification.

OCIS codes: (060.2320) Fiber optics amplifiers and oscillators; (140.3520) Lasers, injection-locked
\end{abstract}

\section{Introduction}

Fiber optic parametric amplifiers (FOPAs) have the potential for noiseless amplification of optical signals when operated in phase-sensitive (PS) mode [1]. One possible implementation of PS amplification is the copier-PSA architecture, consisting of two cascaded FOPAs. The first FOPA, the copier, works as a phase-insensitive amplifier (PIA) generating a phase conjugated copy of the signal at the idler wavelength through four-wave mixing (FWM). The second FOPA, having both signal and idler at input, acts as a phase-sensitive amplifier (PSA).

By inserting a lossy link between the copier and the PSA, it has been shown theoretically [2] and experimentally [3] that up to a $6 \mathrm{~dB}$ noise figure (NF) improvement can be obtained compared to conventional PIA-based amplification schemes, e.g. an erbium-doped fiber amplifier (EDFA)-based scheme. One of the challenges in implementing the copier-PSA scheme in a transmission link is pump recovery, i.e. amplification of the highly attenuated pump. Optical injection-locking (IL) is known to have regenerative features, in particular amplitude squeezing [4], which makes it a promising component in a scheme for achieving pump recovery in a copier-PSA amplified link.

In this paper we demonstrate and characterize, the use of IL for pump recovery in a copier-PSA amplified system for the first time. We compare bit error rate (BER) performance for a PSA-based scheme with PIA- and EDFA-based systems showing a sensitivity advantage for the PSA-based system. For characterization we measured the operation limits for the pump recovery system with and without IL and the standard deviation of the pump amplitude and phase before and after IL.

\section{Experimental setup}

A typical copier-PSA amplified link consists of four basic blocks: copier, transmission link (in our experiment emulated by a loss element), mid-stage, and PSA. After idler generation in the copier, the lossy optical link serves to de-correlate noise on the signal and idler waves [2]. The mid-stage is used for both control of the relative phases, polarizations and amplitudes of the pump, signal, and idler waves and also for recovery of the pump power loss. The waves from the mid-stage are then launched into the PSA stage.

The experimental setup used for characterizing the pump recovery is illustrated in Fig. 1. A 10 Gbaud differential quadrature phase-shift keyed (DQPSK) signal $\left(2^{7}-1\right.$ pseudo-random bit sequence (PRBS)) was modulated onto a wave at $1545.7 \mathrm{~nm}$ with an IQ modulator. A pump wave at $1554.1 \mathrm{~nm}$ was phase modulated with 100 and $300 \mathrm{MHz}$ sinusoids to suppress stimulated Brillouin scattering (SBS) in the parametric amplifiers, broadening the pump FWHM bandwidth to $0.8 \mathrm{GHz}$. After amplification to $7 \mathrm{~W}$ the pump was combined with the signal using a wavelength-division multiplexer (WDM).

The copier consisted of Al-doped highly non-linear fiber (HNLF), providing a net gain of $1 \mathrm{~dB}$, generating an almost power balanced signal/idler pair at the output. After the copier, the waves were fed into the mid-stage where they were split into two branches.

In the mid-stage signal/idler branch, an optical processor (Finisar WaveShaper) was used for filtering, power equalization and attenuation (emulating link loss). The processor was also used to selectively block the idler allowing switching between PS- and PI-mode. An optical delay line matched the optical path length of the signal/idler and pump branches. In the mid-stage pump branch, link loss was emulated with a variable optical attenuator (VOA), VOA1. The pump wave was then pre-amplified using EDFA1 and filtered by a $2 \mathrm{~nm}$ band-pass filter. For IL-based pump recovery the injected power was controlled using VOA2 and the polarization of the injected wave was tuned using a polarization controller (PC), $\mathrm{PC1}$, in order to optimize pump transfer through the IL process. The IL recovered 


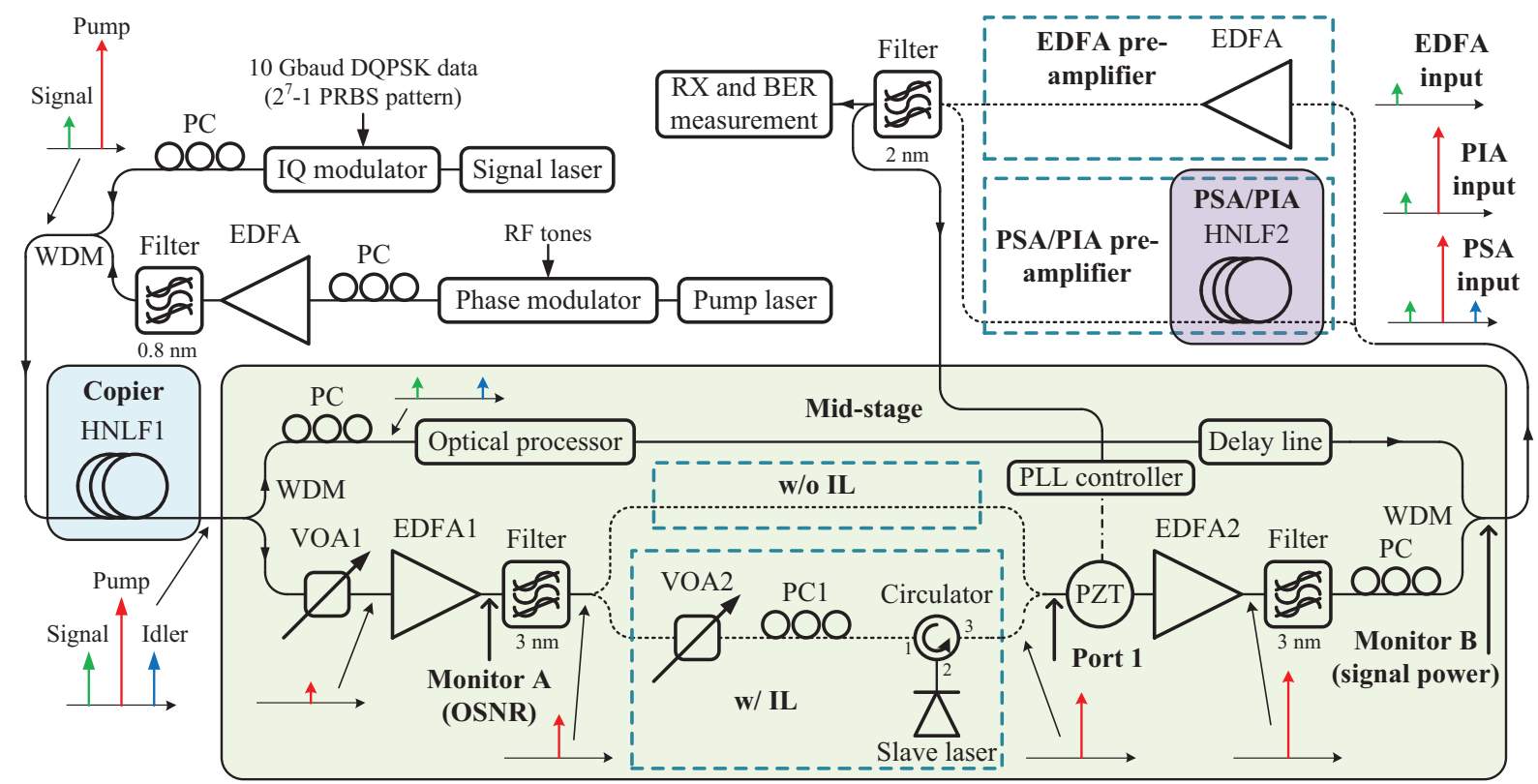

Fig. 1: Experimental setup used for characterization of injection-locking-based pump recovery in a PSA amplified link. Acronyms are explained in the text.

pump was then amplified to $>33 \mathrm{dBm}$ with EDFA2 and filtered. The relative phase between pump signal and idler was kept stable using a piezo-electric transducer (PZT)-based fiber stretcher that was part of a phase-locked loop (PLL).

The final parametric amplifier was implemented with stretched Ge-doped HNLF, with equal signal and idler power launched at input in PS-mode. The gain of the parametric amplifier in both PI- and PS-mode was varied by controlling input pump power to this stage. PCs were used to align the waves before the copier and PSA/PIA.

For the phase and amplitude measurements a coherent detection measurement setup was connected at port 1 . When performing the measurements, the pump EDFA power was reduced and the pump phase modulation was turned off. The standard deviation of the phase and amplitude was measured as a function of the pump optical signal to noise ratio (OSNR) after EDFA1 (monitor A) which was varied by tuning VOA1.

For the BER measurements the received power was measured at monitor B. The pre-amplifier gain was kept at $22 \mathrm{~dB}$ and the received power was varied using the optical processor. The EDFA pre-amplifier was a low-noise preamplifier (JDS Uniphase OAB 1550). For the PIA-case, only the signal and pump were coupled into HNLF2, and for the PSA-case both signal, idler, and pump were coupled. The pre-amplifier output was passed through a filter and then into the receiver (RX), based on interferometer-based differential detection, and the BER measurement system.

\section{Results and discussion}

Measured BER versus received power (signal power at monitor B) is plotted in Fig. 2(a). We compared the PSA preamplifier with the PIA pre-amplifier, both with and without IL in the pump recovery, with the EDFA pre-amplifier. The PSA and PIA pre-amplifiers were compared at three and two different pump OSNRs at monitor A for the case with and without IL, respectively, to characterize the pump recovery.

Fig. 2(a) shows a large performance penalty when decreasing the OSNR from $48 \mathrm{~dB}$ ( $0 \mathrm{dBm}$ at EDFA1 input) to 30 $\mathrm{dB}(-18 \mathrm{dBm}$ at EDFA1 input) in the case without IL, both for the PIA and PSA case. However, when IL is used there is no obvious penalty when reducing the OSNR from $48 \mathrm{~dB}$ down to $18 \mathrm{~dB}$ (-30 dBm at EDFA1 input).

In Fig. 2(b) we plot $Q$ factor penalty against pump OSNR into the IL stage (monitor A), inferring $Q$ from measured BER. The penalty is relative to the $Q$ factor at highest pump OSNR for each system. When using IL in the pump recovery system, the required IL input OSNR for $Q$ factor penalty $<1 \mathrm{~dB}$ is reduced from $\sim 37 \mathrm{~dB}$ without IL to $\sim 8$ $\mathrm{dB}$ with IL. With IL the penalty starts to increase at around $18 \mathrm{~dB}$ of pump OSNR at monitor A.

Fig. 2(a) also shows that both the PSA and PIA pre-amplified receivers demonstrate higher sensitivity than the EDFA pre-amplified receiver when IL-based pump recovery is used. At BER $=10^{-9}$ there is a $5.1 \mathrm{~dB}$ sensitivity improvement of the PSA case compared to the EDFA case and a $0.9 \mathrm{~dB}$ sensitivity improvement with the PIA over the EDFA. Fig. 2(a) also shows that there is a $4.2 \mathrm{~dB}$ sensitivity difference between the PSA case and PIA case. Considering the theoretical $6 \mathrm{~dB}$ lower NF for a PSA amplified system compared to a PIA amplified system [2] the 

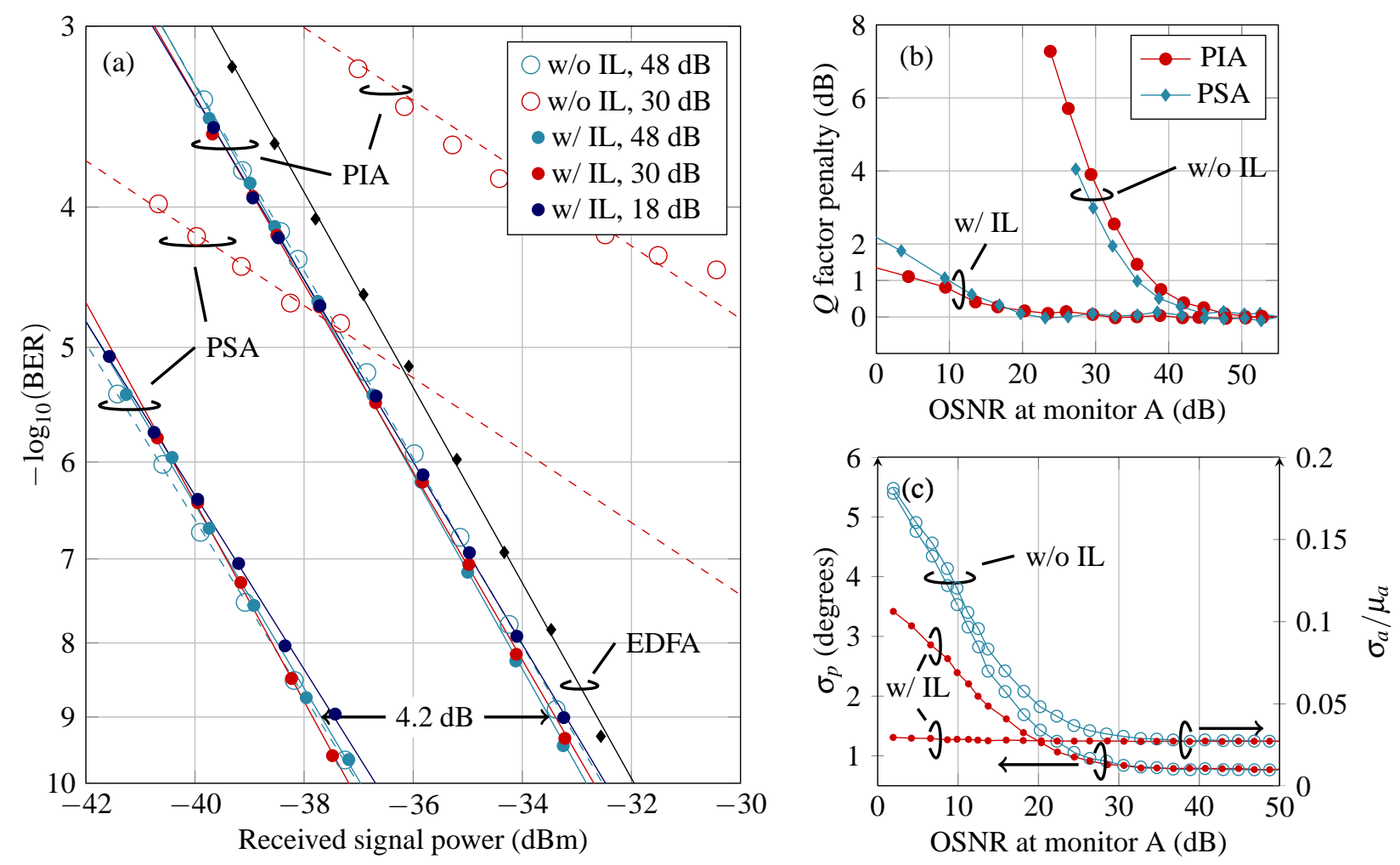

Fig. 2: (a) Measured BER versus received signal power (monitor A) comparing PSA and PIA pre-amplified receivers with and without IL in the pump recovery with an EDFA pre-amplified receiver. Measurements done at different pump OSNR at monitor A, as given in the legend. (b) Q factor penalty versus pump OSNR at monitor A comparing the PSA and PIA case with and without IL. Penalty is relative to the $Q$ factor at highest pump OSNR for each system. (c) Measured amplitude standard deviation over mean $\sigma_{p} / \mu_{p}$ and phase standard deviation $\sigma_{p}$ versus pump OSNR at monitor A. Both amplitude and phase were measured at port 1.

difference may be expected to be higher. However, the difference is the same both with and without IL indicating that there is no significant penalty caused by the IL process itself.

The pump phase standard deviation $\sigma_{p}$ and amplitude standard deviation over mean $\sigma_{a} / \mu_{a}$ at port 1 is plotted in Fig. 2(c). We see that IL suppresses the amplitude noise and to some extent also the phase noise. The reduction of amplitude noise is primarily due to amplitude squeezing. The phase noise reduction is attributed the finite locking bandwidth of the IL laser effectively filtering the broadband ASE noise from EDFA1, reducing both phase and amplitude noise. For each measurement point in Fig. 2(c) VOA1 and PC1 were tuned to ensure good transfer of the pump phase modulation, as when optimized for minimum BER, although the measurements were done with the modulation turned off.

\section{Conclusions}

An IL-based pump recovery system for PSA-amplified links has been proposed and studied experimentally. Recovery of a $0.8 \mathrm{GHz}$ FWHM bandwidth pump with overall pump amplification of $63 \mathrm{~dB}$, from $-30 \mathrm{dBm}$ to $33 \mathrm{dBm}$, is shown to have negligible penalty when measuring BER of 10 Gbaud DQPSK signals transmitted through a copier-PSA amplified system. Coherent measurements show that amplitude noise squeezing and filtering in the IL-process explains the superior performance of IL-based compared to non-IL-based recovery. The benefit of using PSA-amplification compared to PIA-amplification in an amplified receiver arrangement is demonstrated by a $4.2 \mathrm{~dB}$ sensitivity advantage.

\section{Acknowledgements}

The authors would like to thank OFS Denmark for providing the HNLFs. This work was funded by the European Commission STREP project PHASORS (FP7-ICT-2007-2 22457) and by the Swedish Research Council.

\section{References}

[1] C. M. Caves, Quantum limits on noise in linear amplifiers, Phys. Rev. D 26, 1817-1839 (1982).

[2] Z. Tong et al., "Noise performance of optical fiber transmission links that use non-degenerate cascaded phase-sensitive amplifiers," Opt. Express 18, 15426-15439 (2010).

[3] Z. Tong et al., "Towards ultrasensitive optical links enabled by low-noise phase-sensitive amplifiers," Nat. Phot. 5, 430-436 (2011).

[4] A. Furusawa, "Amplitude squeezing of a semiconductor laser with light injection," Opt. Lett. 21, 2014-2016 (1996). 